\title{
COVID-19 herd immunity: where are we?
}

\section{Arnaud Fontanet ${ }^{1,2} \bowtie$ and Simon Cauchemez ${ }^{3 凶}$}

Herd immunity is a key concept for epidemic control. It states that only a proportion of a population needs to be immune (through overcoming natural infection or through vaccination) to an infectious agent for it to stop generating large outbreaks. A key question in the current COVID-19 pandemic is how and when herd immunity can be achieved and at what cost.

Herd immunity is achieved when one infected person in a population generates less than one secondary case on average, which corresponds to the effective reproduction number $R$ (that is, the average number of persons infected by a case) dropping below 1 in the absence of interventions. In a population in which individuals mix homogeneously and are equally susceptible and contagious, $R=\left(1-p_{C}\right)\left(1-p_{I}\right) R_{0}$ (equation 1 ), where $p_{C}$ is the relative reduction in transmission rates due to non-pharmaceutical interventions; $p_{I}$ is the proportion of immune individuals; and $R_{0}$ is the reproduction number in the absence of control measures in a fully susceptible population. $R_{0}$ may vary across populations and over time, depending on the nature and number of contacts among individuals and potentially environmental factors. In the absence of control measures $\left(p_{C}=0\right)$, the condition for herd immunity $\left(R<1\right.$, where $\left.R=\left(1-p_{I}\right) R_{0}\right)$ is therefore achieved when the proportion of immune individuals reaches $p_{I}=1-1 / R_{0}$. For SARS-CoV-2, most estimates of $R_{0}$ are in the range $2.5-4$, with no clear geographical pattern. For $R_{0}=3$, as estimated for France ${ }^{1}$, the herd immunity threshold for SARS-CoV-2 is therefore expected to require $67 \%$ population immunity. It also follows from equation 1 that in the absence of herd immunity, the intensity of social distancing measures necessary to control transmission decreases as population immunity grows. For example, to contain spread for $R_{0}=3$, transmission rates need to be reduced by $67 \%$ if the population is fully susceptible, but by only $50 \%$ if a third of the population is already immune.

There are situations when herd immunity might be achieved before the population immunity reaches $p_{I}=1-1 / R_{0}$. For example, if some individuals are more likely to get infected and to transmit because they have more contacts, these super-spreaders will likely get infected first. As a result, the population of susceptible individuals gets rapidly depleted of these super-spreaders and the pace of transmission slows down. However, it remains difficult to quantify the impact of this phenomenon in the context of COVID-19. For $R_{0}=3$, Britton et al. ${ }^{2}$ showed that, if we account for age-specific contact patterns (for example, individuals aged $>80$ years have substantially less contacts than those aged 20-40 years), the herd immunity threshold drops from $66.7 \%$ to $62.5 \%$. If we further assume that the number of contacts varies substantially between individuals within the same age group, herd immunity could be achieved with only $50 \%$ population immunity. However, in this scenario, the departure from the formula $p_{I}=1-1 / R_{0}$ is only expected if it is always the same set of individuals that are potential super-spreaders. If super-spreading is driven by events rather than by individuals, or if control measures reduce or modify the set of potential super-spreaders, there may be limited impact on herd immunity. Another factor that may feed into a lower herd immunity threshold for COVID-19 is the role of children in viral transmission. Preliminary reports find that children, particularly those younger than 10 years, may be less susceptible and contagious than adults ${ }^{3}$, in which case they may be partially omitted from the computation of herd immunity.

Population immunity is typically estimated through cross-sectional surveys of representative samples using serological tests that measure humoral immunity. Surveys performed in countries affected early during the COVID-19 epidemic, such as Spain and Italy, suggest that nationwide prevalence of antibodies varies between 1 and $10 \%$, with peaks around $10-15 \%$ in heavily affected urban areas ${ }^{4}$. Interestingly, this is consistent with earlier predictions made by mathematical models, using death counts reported in national statistics and estimates of the infection fatality ratio, that is, the probability of death given infection ${ }^{1,5}$. Some have argued that humoral immunity does not capture the full spectrum of SARS-CoV-2 protective immunity and that the first epidemic wave has resulted in higher levels of immunity across the population than measured through cross-sectional antibody surveys. Indeed, T cell reactivity has been documented in the absence of detectable humoral immunity among contacts of patients ${ }^{6}$, although the protective nature and the duration of the observed response are unknown. Another unknown is whether pre-existing immunity to common cold coronaviruses may provide some level of 
cross-protection. Several studies reported cross-reactive $\mathrm{T}$ cells in $20-50 \%$ of SARS-CoV-2-naive individuals ${ }^{7}$. However, whether these T cells can prevent SARS-CoV-2 infection or protect against severe disease remains to be determined ${ }^{7}$. Preliminary reports of surveys in children show no correlation between past infections with seasonal coronaviruses and susceptibility to SARS-CoV-2 infection ${ }^{8}$. Clearly, no sterilizing immunity through cross-protection was evident during the SARS-CoV-2 outbreak on the Charles de Gaulle aircraft carrier, where $70 \%$ of the young adult sailors became infected before the epidemic came to a halt ${ }^{9}$.

Taking these considerations into account, there is little evidence to suggest that the spread of SARS-CoV-2 might stop naturally before at least $50 \%$ of the population has become immune. Another question is what it would take to achieve $50 \%$ population immunity, given that we currently do not know how long naturally acquired immunity to SARS-CoV-2 lasts (immunity to seasonal coronaviruses is usually relatively short lived), particularly among those who had mild forms of disease, and whether it might take several rounds of re-infection before robust immunity is attained. Re-infection has only been conclusively documented in a very limited number of cases so far and it is unclear whether this is a rare phenomenon or may prove to become a common occurrence. Likewise, how a previous infection would affect the course of disease in a re-infection, and whether some level of pre-existing immunity would affect viral shedding and transmissibility, is unknown.

With flu pandemics, herd immunity is usually attained after two to three epidemic waves, each interrupted by the typical seasonality of influenza virus and more rarely by interventions, with the help of cross-protection through immunity to previously encountered influenza viruses, and vaccines when available $^{10}$. For COVID-19, which has an estimated infection fatality ratio of $0.3-1.3 \%^{1,5}$, the cost of reaching herd immunity through natural infection would be very high, especially in the absence of improved patient management and without optimal shielding of individuals at risk of severe complications. Assuming an optimistic herd immunity threshold of $50 \%$, for countries such as France and the USA, this would translate into 100,000-450,000 and 500,000-2,100,000 deaths, respectively. Men, older individuals and those with comorbidities are disproportionally affected, with infection fatality ratios of $3.3 \%$ for those older than 60 years and increased mortality in individuals with diabetes, cardiac disease, chronic respiratory disease or obesity. The expected impact would be substantially smaller in younger populations.

An effective vaccine presents the safest way to reach herd immunity. As of August 2020, six anti-SARS-CoV-2 vaccines have reached phase III trials, so it is conceivable that some will become available by early 2021 , although their safety and efficacy remain to be established. Given that the production and delivery of a vaccine will initially be limited, it will be important to prioritize highly exposed populations and those at risk of severe morbidity. Vaccines are particularly suited for creating herd immunity because their allocation can be specifically targeted to highly exposed populations, such as health-care workers or individuals with frequent contact with customers. Moreover, deaths can be prevented by first targeting highly vulnerable populations, although it is expected that vaccines may not be as efficacious in older people. Vaccines may thus have a significantly greater impact on reducing viral circulation than naturally acquired immunity, especially if it turns out that naturally acquired protective immunity requires boosts through re-infections (if needed, vaccines can be routinely boosted). Also, given that there are increasing numbers of reports of long-term complications even after mild COVID-19, vaccines are likely to provide a safer option for individuals who are not classified at-risk.

For countries in the Northern hemisphere, the coming autumn and winter seasons will be challenging with the likely intensification of viral circulation, as has recently been observed with the return of the cold season in the Southern hemisphere. At this stage, only non-pharmaceutical interventions, such as social distancing, patient isolation, face masks and hand hygiene, have proven effective in controlling the circulation of the virus and should therefore be strictly enforced. Potential antiviral drugs that reduce viral loads and thereby decrease transmission, or therapeutics that prevent complications and deaths, may become significant for epidemic control in the coming months. This is until vaccines become available, which will allow us to reach herd immunity in the safest possible way.

1. Salje, H. et al. Estimating the burden of SARS-CoV-2 in France. Science 369, 208-211 (2020).

2. Britton, T., Ball, F. \& Trapman, P. A mathematical model reveals the influence of population heterogeneity on herd immunity to SARS-CoV-2. Science 369, 846-849 (2020).

3. Goldstein, E., Lipsitch, M. \& Cevik, M. On the effect of age on the transmission of SARS-CoV- 2 in households, schools and the community. Preprint at medRxiv https://doi.org/10.1101/ 2020.07.19.20157362 (2020).

4. Byambasuren, O. et al. Estimating the seroprevalence of SARS-CoV-2 infections: systematic review. Preprint at medRxiv https://doi.org/10.1101/2020.07.13.20153163 (2020).

5. Flaxman, S. et al. Estimating the effects of non-pharmaceutical interventions on COVID-19 in Europe. Nature 584, 257-261 (2020).

6. Sekine, T. et al. Robust T cell immunity in convalescent individuals with asymptomatic or mild COVID-19. Cell https://doi.org/10.1016 j.cell.2020.08.017 (2020).

7. Sette, A. \& Crotty, S. Pre-existing immunity to SARS-CoV-2: the knowns and unknowns. Nat. Rev. Immunol. 20, 457-458 (2020).

8. Sermet-Gaudelus, I. et al. Prior infection by seasonal coronaviruses does not prevent SARS-CoV-2 infection and associated multisystem inflammatory syndrome in children. Preprint at medRxiv https://doi.org/10.1101/2020.06.29.20142596 (2020)

9. Service de santé des armées. Investigation de l'épidémie de COVID-19 au sein du Groupe Aéronaval. Service de santé des armées https://www.defense.gouv.fr/content/download/583466/9938746 file/20200405_929_ARM_SSA_CESPA_rapport_epidemie_covid 19 Gan VEXP.pdf (2020)

10. Miller, M. A., Viboud, C., Balinska, M. \& Simonsen, S. The signature features of influenza pandemics - implications for policy. N. Engl. J. Med. 360, 2595-2598 (2009).

Competing interests

A.F. and S.C. are members of the COVID-19 Scientific Council advising the French Ministry of Health. 